Research Article

\title{
Mixed-Integer Linear Programming Model by Linear Approximation for a Strike Package-to-Target Assignment Problem
}

\author{
Heungseob Kim (1) \\ Department of Industrial and Systems Engineering, Department of Smart Manufacturing Engineering, \\ Changwon National University, Changwon, Republic of Korea
}

Correspondence should be addressed to Heungseob Kim; heungseob79@cwnu.ac.kr

Received 11 December 2020; Revised 21 March 2021; Accepted 7 April 2021; Published 21 April 2021

Academic Editor: Qingzheng XU

Copyright (c) 2021 Heungseob Kim. This is an open access article distributed under the Creative Commons Attribution License, which permits unrestricted use, distribution, and reproduction in any medium, provided the original work is properly cited.

\begin{abstract}
This study deals with an aircraft-to-target assignment (ATA) problem considering the modern air operation environment, such as the strike package concept, multiple targets for a sortie, and the strike packages' survivability. For the ATA problem, this study introduces a novel mathematical model in which a heterogeneous vehicle routing problem (HVRP) and a weapon-to-target assignment (WTA) problem are conceptually integrated. The HVRP generates the flight routes for strike packages because this study confirms that the survivability of a strike package depends on the path, and the WTA problem evaluates the likelihood of successful target destruction of assigned weapons. Although the first version of the model is developed as a mixed-integer nonlinear programming (MINLP) model, this study attempts to convert it to a mixed-integer linear programming (MILP) model using the logarithmic transformation and piecewise linear approximation methods. For an ATA problem, this activity could provide an opportunity to use the excellent existing algorithms for searching the optimal solution of LP models. To maximize the operational effectiveness, the MILP model simultaneously determines the following for each strike package: (a) composition type, (b) targets, (c) flight route, (d) types, and (e) quantity of weapons for each target.
\end{abstract}

\section{Introduction}

The advancement of weapon systems has led to a change in the paradigm of war in the manner of operations. The representative conceptions of modern warfare are defined as network-centric warfare (NCW), which shares the information on an overall battlefield in real time by integrating information collection systems, and an effect-based operation (EBO) aims to achieve war objectives early by surgically striking only the core targets. The weapon-to-target assignment (WTA) problem, which is one of the major research topics in the field of operations research and management science (OR/MS), has been continuously studied to meet the modern battlefield environment. It is also a certain case of the general resource allocation problem in industrial engineering.

The WTA problem was proposed in 1951 by Merrill M. Flood to allocate multiple weapons to multiple targets to save ammunition and maximize operational effects. After that, research has been actively conducted based on a model proposed by Manne [1], which improved the function for evaluating the operational effectiveness. Although most of the researches on WTA have focused on combat concepts for ground forces, studies that applied it to air defense operations [2-4], maritime operations $[5,6]$, and UAVs combat system $[7,8]$ have appeared recently.

The WTA problem for air operations is also called the aircraft-to-target assignment (ATA) problem, and it was first suggested by Wegner [9] as a special case of the weighted distribution problem. An ATA problem, which introduced the notion of a strike package applied in modern warfare, was first proposed by Griggs [10]. A strike package is a group of aircraft with different capabilities launched together to perform a single-attack mission. It includes bombers to attack a primary target, fighters to safeguard them from enemy aircraft, wild weasels to defend against ground-based 
antiaircraft weapons, reconnaissance aircraft for preraid and postraid reconnaissance, and tanker aircraft to extend the mission radius. The most significant difference between the ATA and the traditional WTA problem is that the enemy may assault the aircraft because they should execute operations in hostile territory. In other words, the ATA problem considers not only the probability of kill against a target but also the survival probability of a strike package.

The subject of existing studies on the ATA problem is divided into assigning a sortie for a target, that is, sortie-totarget assignment, and assigning a sortie for some targets, that is, targets-to-sortie assignment $[11,12]$. The sortie-totarget assignment problem assumes that a sortie can attack only one target, similar to the WTA problem [13-15]. However, in modern warfare, where Precision-Guided Munitions (PGMs) are used, a sortie of a fighter can strike multiple targets. In this context, Rosenberger et al. [16] proposed the targets-to-sortie assignment problem by extending the basic WTA. Based on the work of Rosenberger et al. [16], Lee and Yang [12] have suggested a mixed-integer programming (MIP) model that reduced the feasible solution search space. Ahner and Parson [17] and Parson [18] presented an approximate dynamic programming model.

Furthermore, studies on the solution methodologies for a WTA have been actively performed because Lloyd and Witsenhausen [19] proved that it is a combinatorial optimization problem and is NP-Complete. Some researches, such as those of Chang et al. [20], Orlin [21], and Lee and Shin [22], suggested exact solution methods under the assumption of one type of weapon or less than one bomb per target. Kwon et al. [23] linearized the WTA model by the logarithmic transformation and proposed the Lagrangian relaxation approach and branch-andbound (B\&B) algorithm. Ahuja et al. [24] considered the WTA as a network flow model and suggested the B\&B method and neighborhood search-based heuristic method for searching lower and upper bounds individually. In recent years, metaheuristic algorithms have been developed, such as neural network [25], genetic algorithm $[6,26-28]$, ant colony optimization $[29,30]$, tabu search [31], stable marriage algorithm [32], and variable neighborhood search [33]. Moreover, hybrid algorithms combining two or more other algorithms have also been tried [34-36].

This study suggests an advanced targets-to-sortie model for establishing a more realistic and reasonable air operations plan. Existing studies determined combinations of "strike package-to-targets" and "weapon-to-target" $[11,12,16]$. However, for individual strike packages, the model proposed in this study simultaneously provides information on the composition type of a strike package, its flight route, and the type and quantity of the weapon for attacking targets assigned to it. Furthermore, because the previous studies considered that the survival probability of a strike package flying between any two targets is independent of a former route, the flight route is unimportant to them. However, the survivability for a strike package that performs attacks on multiple targets should be evaluated considering overall threats on the planned flight route. This research has confirmed that it depends on the order of attack on the assigned targets (see Section 2.1). Thus, the suggested model is formulated by integrating the ATA problem and the heterogeneous vehicle routing problem (HVRP). The original problem is developed as a mixedinteger nonlinear programming (MINLP) model because the objective function and some constraints are represented in nonlinear form. Thus, this study attempts to linearize them using the logarithmic transformation and piecewise approximation.

The rest of the article is organized as follows. Section 2 reviews the formulation for evaluating the operational effectiveness and presents the mathematical model to establish the operation plan (OPLAN) to maximize the effectiveness. Section 3 suggests the linearization methods for nonlinear functions included in the model in Section 2. Section 4 provides the results of numerical experiments for verifying and validating the mathematical model. Section 5 provides the conclusion and future research directions.

\section{Problem Statement}

2.1. Operational Effectiveness. In traditional WTA problems, the mission success indicator (MSI) for a target depends on the assigned weapon. The MSI for target $i$ has been represented as a function with regard to its value $\left(V_{i}\right)$ and probability $\left(p_{w i}\right)$ that it will be destroyed by the quantity $\left(y_{w i}\right)$ of weapon $w$. Namely, given the probability that $\left(p_{w i}\right)$ will destroy target $i$ with a single shot of weapon $w$, $\mathrm{MSI}_{i}=V_{i}\left[1-\left(1-p_{w i}\right)^{y_{w i}}\right]$. In this study, the operational effectiveness is defined as the sum of the MSIs for individual targets.

However, in air operations, fighter aircraft's survivability has to be considered because they perform the mission at the battlefield where the enemy threat always resides. Existing studies have separately considered survival probabilities between each of the two targets on a flight route containing multiple targets. That is, when estimating the MSI for the second target, it is assumed that the fighter survived in the mission for the first target. For example, in Figure 1, it is considered that $\left(p_{w i}=1.0\right)$ for $(i \in 1,2)$, and $P\left(\overline{T_{i} T_{j}}\right)$ denotes the survival probability on flight path $\left(\overline{T_{i} T_{j}}\right)$. When a strike package takes off from the base $T_{0}$ to attack targets $T_{1}$ and $T_{2}$, its flight path may be $\theta_{1}=\overline{T_{0} T_{1} T_{2}}$ or $\theta_{2}=\overline{T_{0} T_{2} T_{1}}$. By the previous method, the operational effectiveness on flight routes $\theta_{1}$ and $\theta_{2}$ is equally evaluated as $Z_{1}\left(\theta_{1}\right)=\left[V_{1} P\left(\overline{T_{0}} T_{1}\right)+\right.$ $\left.V_{2} P\left(\overline{T_{1} T_{2}}\right)\right]=2.7$ and $Z_{1}\left(\theta_{2}\right)=\left[V_{2} P\left(\overline{T_{0} T_{2}}\right)+V_{1} P\left(\overline{T_{2} T_{1}}\right)\right]$ $=2.7$, respectively. However, because the strike package should survive on the paths $\left(\overline{T_{0} T_{1}}\right)$ and $\left(\overline{T_{1} T_{2}}\right)$ to get a chance for attacking target $T_{2}$, the assumption of the previous method is unreasonable. In other words, when $\theta_{1}=\overline{T_{0} T_{1} T_{2}}$, it is reasonable to estimate the operational effectiveness as $Z_{2}\left(\theta_{1}\right)=\left[V_{1} P\left(\overline{T_{0} T_{1}}\right)+V_{2} P\left(\overline{T_{0} T_{1}}\right) P\left(\overline{T_{1} T_{2}}\right)\right]=2.52$. Moreover, considering the proposed concept, the optimal flight path is $\theta_{2}=\overline{T_{0} T_{2} T_{1}}$ with $Z_{2}\left(\theta_{2}\right)=2.61$. Finally, the existing studies have considered that the flight route, including the sequence of an attack on the targets, is independent of the operational effectiveness. However, the proposed concept 


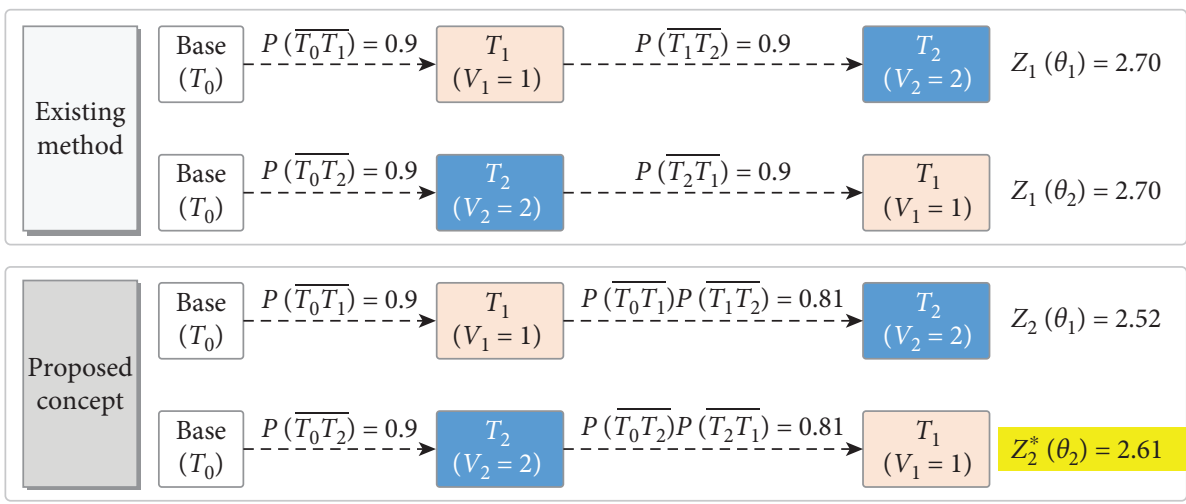

FIGURE 1: Comparison of the methods for evaluating operational effectiveness.

confirms that the flight route is an essential factor for a realistic and reasonable OPLAN.

Finally, according to the proposed method, the operational effectiveness is expressed as a nonlinear function $f(\mathbf{V}, \boldsymbol{\theta}, \mathbf{P}, \mathbf{p})$ with regard to some vectors for values $\mathbf{V}$ of targets, the flight routes $\boldsymbol{\theta}$ and survival probabilities $\mathbf{P}$ of a strike package at each target, and the destructive probabilities, $p=g\left(p_{w}, y_{w}\right)$, of targets. For example, when the flight route of a strike package is determined by $\theta_{1}=\overline{T_{0} T_{1} T_{2}}$, the operational effectiveness $f\left(\mathbf{V}, \mathbf{P}, \mathbf{p} \mid \theta_{1}\right)$ is estimated as shown in Table 1.

2.2. Mixed-Integer Nonlinear Programming (MINLP) Model. For decision-making support in air operations, this section introduces the mixed-integer nonlinear programming (MINLP) model with a nonlinear objective function, and we make the following assumptions: (1) Strike packages consist of multiple aircraft for various missions, such as attack, electronic warfare, fighter escort, suppression of enemy air defense (SEAD), command control, communications, and battle damage assessment (BDA) [37]. The compositions will be reviewed and determined in advance and affect operational effectiveness due to the variation of survivability and the destructive probability of targets. (2) Each aircraft in a strike package is equipped with a predetermined weapon set according to its mission. (3) To attack a target, heterogeneous weapons could be used, and the probability of the target being killed depends on both the type and quantity of assigned weapons.

The MINLP model is mathematically expressed as equations (1)-(20), and the notations and decision variables are summarized in Table 2. To maximize the effectiveness of overall operations, it simultaneously determines the following for each strike package: (a) composition type, (b) targets, (c) flight route, (d) types, and (e) quantity of weapons to attack the targets.

maximize $\sum_{i \in \mathbf{T}} V_{i} \cdot C P_{i} \cdot\left[1-\prod_{w \in \mathbf{W}}\left(1-p_{w i}\right)^{y_{w i}}\right]$,

subject to

$$
\begin{aligned}
& \sum_{s \in \mathbf{S}} \sum_{i \in \mathbf{N}(i \neq j)} x_{s i j}=1, \quad \forall j \in \mathbf{T}, \\
& \sum_{i \in \mathbf{N}} x_{s i p}-\sum_{j \in \mathbf{N}} x_{s p j}=0, \quad \forall s \in \mathbf{S}, p \in \mathbf{N}, \\
& x_{s i i}=0, \quad \forall s \in \mathbf{S}, i \in \mathbf{T}, \\
& u_{i}-u_{j}+|\mathbf{N}| x_{s i j} \leq|\mathbf{N}|-1, \quad \forall s \in \mathbf{S}, i, j \in \mathbf{T}(i \neq j), \\
& t_{s j} \geq t_{s i}+\left(O_{s i}+\frac{d_{i j}}{v_{s}}\right) x_{s i j}-M\left(1-x_{s i j}\right), \\
& t_{s i}+\frac{d_{i 0}}{v_{s}} x_{s i 0} \leq \overline{F T_{s}}, \quad \forall s \in \mathbf{S}, i \in \mathbf{N}, j \in \mathbf{T}(j \neq i), \\
& C P_{0}=1.0,
\end{aligned}
$$$$
C P_{j} \leq C P_{i} \cdot P_{s i j}+\left(1-x_{s i j}\right), \quad \forall s \in \mathbf{S}, i \in \mathbf{N}, j \in \mathbf{T}(i \neq j),
$$$$
\overline{W_{j}} \sum_{s \in \mathbf{S}} \sum_{i \in \mathbf{N}} x_{s i j}-\sum_{w \in \mathbf{W}} y_{w j} \geq 0, \quad \forall j \in \mathbf{T},
$$

$z_{s w j} \geq z_{s w i}+y_{w j}-M\left(1-x_{s i j}\right), \quad \forall s \in \mathbf{S}, i \in \mathbf{N}, j \in \mathbf{T}(j \neq i)$,

$$
z_{s w j} \leq q_{s w} \sum_{i \in \mathbf{N}(i \neq j)} x_{s i j}, \quad \forall s \in \mathbf{S}, w \in \mathbf{W}, j \in \mathbf{T},
$$

$\sum_{s \in \mathbf{S}} \sum_{j \in \mathbf{T}} J_{s k} x_{s 0 j} \leq \overline{S T_{k}}, \quad \forall k \in \mathbf{K}$,

$\sum_{i \in \mathbf{T}} y_{w i} \leq \overline{Q_{w}}, \quad \forall w \in \mathbf{W}$,

$x_{s i j} \in\{0,1\}, \quad \forall s \in \mathbf{S}, i, j \in \mathbf{N}$,

$y_{w i} \in \mathbb{Z}^{+}, \quad \forall w \in \mathbf{W}, i \in \mathbf{T}$, 
TABLE 1: Example of calculating the operational effectiveness.

\begin{tabular}{lc}
\hline Target & Operational effectiveness \\
\hline T1 & $f_{1}\left(V_{1}, P_{1}, p_{1} \mid \theta_{1}\right)=V_{1} P\left(\overline{T_{0} T_{1}}\right)\left[1-\prod_{w \in \mathbf{W}}\left(1-p_{w 1}\right)^{y_{w 1}}\right]$ \\
T2 & $f_{2}\left(V_{2}, P_{2}, p_{2} \mid \theta_{1}\right)=V_{2}\left[P\left(\bar{T}_{0} T_{1}\right) P\left(\overline{T_{1} T_{2}}\right)\right]\left[1-\prod_{w \in \mathbf{W}}\left(1-p_{w 2}\right)^{y_{w 2}}\right]$ \\
Total & $f\left(\mathbf{V}, \mathbf{P}, \mathbf{p} \mid \theta_{1}\right)=f_{1}\left(V_{1}, P_{1}, p_{1} \mid \theta_{1}\right)+f_{2}\left(V_{2}, P_{2}, p_{2} \mid \theta_{1}\right)$ \\
\hline
\end{tabular}

TABLE 2: Notations for the mathematical model.

\begin{tabular}{|c|c|}
\hline Indices & Description sets \\
\hline $\begin{array}{l}\text { S } \\
\text { W } \\
\text { T } \\
\text { N } \\
\text { K }\end{array}$ & $\begin{array}{c}\text { Set of strike package types; } s \in \mathbf{S} \\
\text { Set of weapon types; } w \in \mathbf{W} \\
\text { Set of target nodes } \\
\text { Set of base }\{0\} \text { and target nodes; } \mathbf{N}=\{0\} \cup \mathbf{T} \\
\text { Set of aircraft types; } k \in \mathbf{K}\end{array}$ \\
\hline $\begin{array}{l}\text { Parameters } \\
\left(V_{i}\right) \\
p_{s i j} \\
p_{w i} \\
d_{i j} \\
\frac{v_{s}}{F T_{s}} \\
O_{s i} \\
\frac{J_{s k}}{S_{k}} \\
\overline{W_{i}} \\
\frac{q_{s w}}{Q_{w}} \\
\mathrm{M}\end{array}$ & $\begin{array}{c}\text { Value of target } i \\
\text { Probability of survival against enemy threat between nodes } i \text { and } j \\
\text { Probability of destroying target } i \text { with weapon } w \\
\text { Distance between nodes } i \text { and } j \\
\text { Flight speed of strike package } s \\
\text { Maximum flight time of strike package type } s \\
\text { Offensive mission time for target } i \text { of strike package } s \\
\text { Number of aircraft type } k \text { joining in strike package type } s \\
\text { Maximum sorties of aircraft type } k \\
\text { Maximum number of weapons for attacking target } i \\
\text { Amount of loaded weapon } w \text { on strike package } s \\
\text { Total number of weapons } w \text { for the air operation } \\
\text { Sufficiently large number (Big-M) }\end{array}$ \\
\hline $\begin{array}{l}\text { Decision variables } \\
x_{s i j}= \\
y_{w i} \\
z_{\text {swi }} \\
t_{s i} \\
C P_{i} \\
u_{i}\end{array}$ & 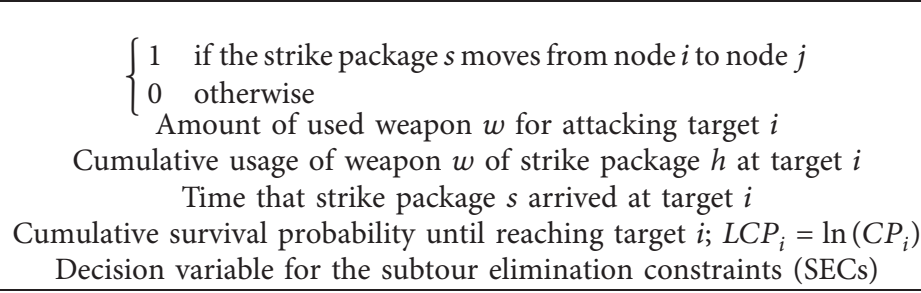 \\
\hline
\end{tabular}

$$
\begin{aligned}
& z_{s w i} \in \mathbb{Z}^{+}, \forall s \in \mathbf{S}, w \in \mathbf{W}, i \in \mathbf{N}, \\
& t_{s i} \in \mathbb{R}^{+}, \forall s \in \mathbf{S}, i \in \mathbf{N}, \\
& C P_{i} \in \mathbb{R}^{+}, \quad C P_{i} \leq 1, \forall i \in \mathbf{N}, \\
& u_{i} \in \mathbb{R}^{+}, \quad \forall i \in \mathbf{N} .
\end{aligned}
$$

The objective function, equation (1), is the operational effectiveness, that is, reward accumulated by attacking all targets, and it is the generalized form of the function $f(\mathbf{V}, \boldsymbol{\theta}, \mathbf{P}, \mathbf{p})$ suggested in Section 2.1. However, it has nonlinearity for the following reasons: First, it is represented as the multiplication form of terms involving the decision variables $C P_{i}$ and $y_{w i}$. Second, the term for evaluating the $\mathrm{MSI}_{i}$ for target $i$ is expressed as a $\sum_{w \in \mathbf{W}} y_{w i}$-order polynomial function. This study will convert the MINLP model to an approximate mixed-integer linear programming (MILP) model, and the methodologies for linearizing the MINLP model are presented in Section 3.

Constraints (2)-(5) are to assign targets to strike packages and create their flight routes. Constraint (2) guarantees that each target is assigned to only one strike package. Constraint (3) ensures the flight route's continuity for individual strike packages, and constraint (4) means that the packages do not stay at a target. Constraints (5) are subtour elimination constraints (SECs) that are used to preclude infeasible solutions involving incomplete routes disconnected from the base, as shown in Figure 2(a) $[38,39]$. In other words, if a set of decision variables $x$ sij generates a flight route containing subtours, there will be no $u_{i}$ that simultaneously satisfies the SECs for each target node.

Equations (6) and (7) are constraints related to flight times of strike packages, and constraint (6) calculates the time when the package assigned targets $i$ and $j$ arrives at target $j$ after attacking target $i$. Constraint (7) limits the total flight time of strike package $s$, including the time to return to base $\{0\}$ (RTB). As shown in Figure 2(b), it states that when package $s$ arrives at target $i$, the residual flight time should be able to ensure RTB.

Constraint (8) designates the survival probability of packages taking off from the base as 1.0, and constraint (9) determines the cumulative survival probability of consecutive targets in each flight route. Equations (10)-(12) provide constraints for assigning weapons to targets. Constraint (10) 


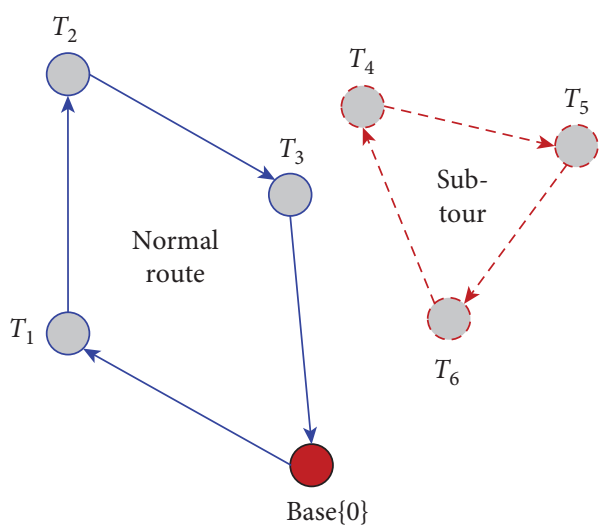

(a)

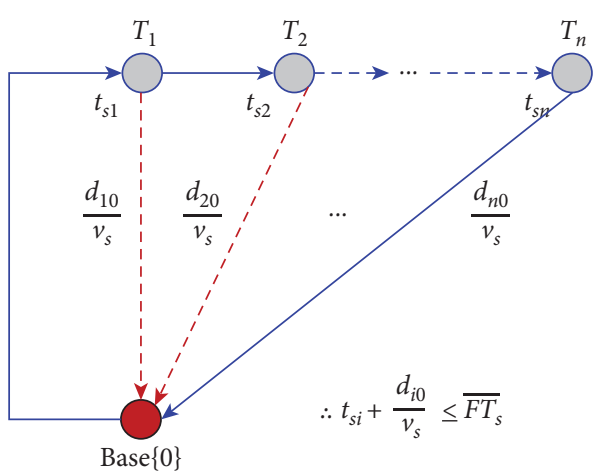

(b)

Figure 2: Schematic explanation of equations (5) and (7). (a) Normal and subtour routes. (b) Flight time to ensure RTB.

associates the weapon-target assignment with the flight route of a strike package. It specifies that a package can only attack targets on its flight route and limits the maximum number of weapons used for a target. Constraint (11) determines the accumulated number of weapons $w$ fired on consecutive targets along a flight path, and constraint (12) specifies that the aggregate of weapon $w$ used by package $s$ cannot exceed the quantity loaded on it. For overall operations, constraints (13) and (14) stipulate the upper limits of the total sorties of each aircraft type and the total amount of each weapon, respectively. Finally, constraints (15)-(20) designate the type and range of the decision variables, $x_{s i j}, y_{w i}, z_{s w i}, t_{s i}, C P_{i}$, and $u_{i}$, respectively.

\section{Linearization of the Proposed MINLP Model}

The MINLP model suggested in Section 2.2 has a nonlinear objective function, equation (1). In this section, the process for converting the MINLP to a mixed-integer linear programming (MILP) model is presented. A logarithmic transformation and piecewise linear approximation methods are used in the process. By linearizing the objective function, constraints for estimating the survival probability of a strike package at a target, equations (8) and (9), should also be modified. The transformation processes for the objective function and constraints are discussed in Sections 3.1 and 3.2, respectively.

3.1. Objective Function: Equation (1). The most significant nonlinearity of the objective function, equation (1), is that it is represented as the multiplication form of terms involving the decision variables $C P_{i}$ and $y_{w i}$. Thus, to separate the terms for them and convert them into a linear combination form, a logarithmic transformation is introduced. The logarithmic transformation of the MSI of each target gives (21), providing the equivalent purpose as equation (1), where $L C P_{i}=\ln \left(C P_{i}\right)$, and $\mathrm{NDP}_{i}$ denotes the probability that target $i$ will be not destroyed in the attack of a strike package. That is, $\mathrm{NDP}_{i}=\prod_{w \in \mathrm{W}}\left(1-p_{w i}\right)^{y_{w i}} . \quad L C P_{i}$ requires the modification of constraints (8) and (9), and they are presented in Section 3.2. Moreover, a methodology for linearly approximating $\ln \left(1-\mathrm{NDP}_{i}\right)$ is introduced in Section 3.3.

$$
\sum_{i \in \mathbf{T}}\left[\ln V_{i}+L C P_{i}+\ln \left(1-\mathrm{NDP}_{i}\right)\right]
$$

3.2. Constraints for Evaluating the Survivability: Equations (8) and (9). To estimate $L C P_{i}$ generated by converting the objective function in Section 3.1, equations (8) and (9) in the MINLP model suggested in Section 2.2 should also be modified. Equation (8) to specify the initial survival probability of strike packages is replaced by equation (22) because $\ln \left(C P_{0}=1.0\right)=0$. Moreover, equation (9) for estimating the cumulative survival probability $L C P_{i}$ at target $i$ for a strike package should also be replaced by equation (23). Herein, the term $M\left(1-x_{s i j}\right)$ functions to sufficiently open the feasible range of $L C P_{j}$ when strike package $s$ does not fly from node $i$ to $j$; that is, $x_{s i j}=0$.

$$
\begin{aligned}
& L C P_{0}=0, \\
& L C P_{j} \leq L C P_{i}+\ln P_{s i j}+\mathrm{M}\left(1-x_{s i j}\right), \\
& \forall s \in \mathbf{S}, i \in \mathbf{N}, j \in \mathbf{T}(i \neq j) .
\end{aligned}
$$

3.3. Term $\ln \left(1-N D P_{i}\right)$ in Equation (21). To linearize the term $\ln \left(1-\mathrm{NDP}_{i}\right)$, which is still nonlinear, generated by converting the objective function in Section 3.1, the piecewise linear approximation is introduced. Furthermore, $\left(\mathrm{NDP}_{i}\right)$ is transformed as shown in equation (24), and the piecewise approximation constraints are applied to the relationship between equation (24) and the term, $f(\eta)=\ln \left(1-\mathrm{NDP}_{i}\right)$ where $\eta=\ln \left(\mathrm{NDP}_{i}\right)$, in equation (21).

$$
\ln \mathrm{NDP}_{i}=\ln \prod_{w \in \mathbf{W}}\left(1-p_{w i}\right)^{y_{w i}}=\sum_{w \in \mathbf{W}} y_{w i} \ln \left(1-p_{w i}\right) .
$$

To manage the piecewise approximation error within a reasonable level, the appropriate numbers of intervals and 
breakpoints should be determined. The notations used for a piecewise linear approximation model, as shown in Figure 3, are summarized in Table 3. This study employs a simple multivariate nonlinear optimization model, equation (25), for finding the optimal breakpoints $\left(\eta_{1} \equiv \mathrm{NDP}_{\mathrm{i}}\right)$ in search space $-\infty, 0$, when the number of intervals is given as $L$. In interval $\left[\eta_{l}, \eta_{l}+1\right)$, as shown in Figure 3, the approximation error $\delta_{\left[\eta_{l}, \eta_{l}+1\right)}(\eta)$ is maximized at point $\eta_{\left[\eta_{l}, \eta_{l}+1\right)}^{*}$, and the point is represented as equation (26). Therefore, the objective function of the model is to minimize the largest value of the maximum errors, $\delta_{\left[\eta_{l}, \eta_{l}+1\right)}^{*}=f\left(\eta_{\left[\eta_{l}, \eta_{l}+1\right)}^{*}\right)-$ $g_{\left[\eta_{l}, \eta_{l}+1\right)}\left(\eta_{\left[\eta_{l}, \eta_{l}+1\right)}^{*}\right)$, in individual intervals, and the errors are always positive because $f(\eta) \geq g(\eta)$ for all $\eta$. Finally, in interval $\left[\eta_{l}, \eta_{l}+1\right)$, the piecewise linear approximation constraint for term $\ln \left(1-\mathrm{NDP}_{i}\right)$ in equation (21) is expressed as equation (27) because $f(-\infty)=0$, and it should be added to the MINLP model proposed in Section 2.2 .

$$
\begin{aligned}
\text { Minimizemax }\left[\max \left\{\delta_{\left[\eta_{l}, \eta_{l+1}\right)}(\eta)\right\} ; l=0,1, \ldots, L\right]=\max \left[\delta_{\left[\eta_{l}, \eta_{l+1}\right)}^{*}\right], & \\
\text { subject to : } \quad & \eta_{l} \leq \eta_{l+1}, 0 \leq l \leq L, \\
& -\infty<\eta_{l} \leq 0,0 \leq l \leq L, \\
\eta_{\left[\eta_{l}, \eta_{l+1}\right)=}^{*} & \ln \left(\frac{\alpha_{\left[\eta_{l}, \eta_{l+1}\right)}}{\alpha_{\left[\eta_{l}, \eta_{l+1}\right)}-1}\right)=\ln \left[\frac{e^{\eta_{l}}-e^{\eta_{l+1}}}{\left(e^{\eta_{l}}-e^{\eta_{l+1}}-\left(\eta_{l+1}-\eta_{l}\right)\right.}\right], \\
\ln \left(1-\mathrm{NDP}_{i}\right)= & {\left[\sum_{n=0}^{l-1} \alpha_{\left[\eta_{l}, \eta_{l+1}\right)}\left(\eta_{n+1}-\eta_{n}\right)\right]+\alpha_{\left[\eta_{l}, \eta_{l+1}\right)}\left(\eta-\eta_{l}\right), \quad \eta_{l} \leq \eta<\eta_{l+1} . }
\end{aligned}
$$

For example, when the range of the NDP is set to 10-6, 0.5 , that is, $(1-\mathrm{NDP}) \in[0.5,0.999999]$, and the number of intervals was considered from 2 to 25 , the maximum approximation error decreases with increasing number of intervals, as shown in Figure 4(a). Piecewise linear approximation models with 5, 10, and 20 intervals are shown in Figures 4(b)-4(d), respectively. Furthermore, the optimal breakpoints $\left(\eta_{l}\right)$ and slopes $\left(\alpha_{\left[\eta_{l}, \eta_{l}+1\right)}\right)$ for the models with 10,15 , and 20 intervals are presented in Table 4. Here, though the $\left(1-\mathrm{NDP}_{i}\right)$ range has been set relatively wide, its narrow range, considering the precision of modern weapons, contributes to reducing the maximum approximation error as shown in Figure 5.

\section{Numerical Experiments}

Numerical experiments are performed to verify the mathematical model introduced in Sections 2 and 3 and evaluate the accuracy of the piecewise linear approximation. The experiments were performed using ILOG CPLEX Ver. 12.8 in a $2.5 \mathrm{GHz}$ Intel Core i7-6500U CPU and $16 \mathrm{~GB}$ RAM environment.

4.1. Experimental Example. Numerical experiments are performed on a simple example to analyze the performance error caused by the linear approximation. The breakpoints for the piecewise linear function with 10 and 15 intervals are shown in Table 4. The example is establishing an air operation to attack ten targets, and the input parameters are presented in Table 5. The target coordinates were randomly set in the front area with a friendly base as the origin $(0,0)$. The $\mathrm{J}_{s k}$ and $\mathrm{q}_{s w}$ matrices are described as follows: The strike packages consist of four aircraft and eight weapons. For example, the first strike package $(s=1)$ includes two aircraft, each of the first and second types $(k=1$ and $k=2)$, and it can use up to 4 weapons, each of the first and second types ( $w=1$ and $w=2$ ). Each aircraft type's resources have an upper limit of 8 sorties, and weapons for attacking a target are allocated within three. Besides, the survival probability $P_{s i j}$ of a strike package $(s)$ flying from target $i$ to $j$ is considered to be inversely proportional to the distance $d_{i j}$ between the two targets, such as $H_{s} /\left(H_{s}+d_{i j}\right)[15]$.

4.2. Experimental Results. Based on the input parameters presented in Table 5, numerical experiments are performed on MILP models with piecewise linear approximation functions with 10 and 15 intervals. The computational times for them were 128.3 and 142.8 seconds, respectively. The strike-package types and each package's flight route, including targets assigned to the strike package and attack sequence, were output identically, as shown in Figure 6. However, as shown in Table 6, there are differences in the weapons assigned to several targets, and the difference is due to the dissimilar errors of the approximation methods.

The numerical analysis of the error of the approximation methods is presented in Table 7 . The real values in Table 7 were recalculated by equation (1) based on the optimal solutions of the MILP models. Due to the approximation error of piecewise linear function for estimating 1-NDPi, there is a subtle deviation of the MSIs at targets. In the numerical experiment applying the piecewise linear function with 10 intervals, the objective function's error is evaluated as $0.67 \%$, and the error of MSI at the 


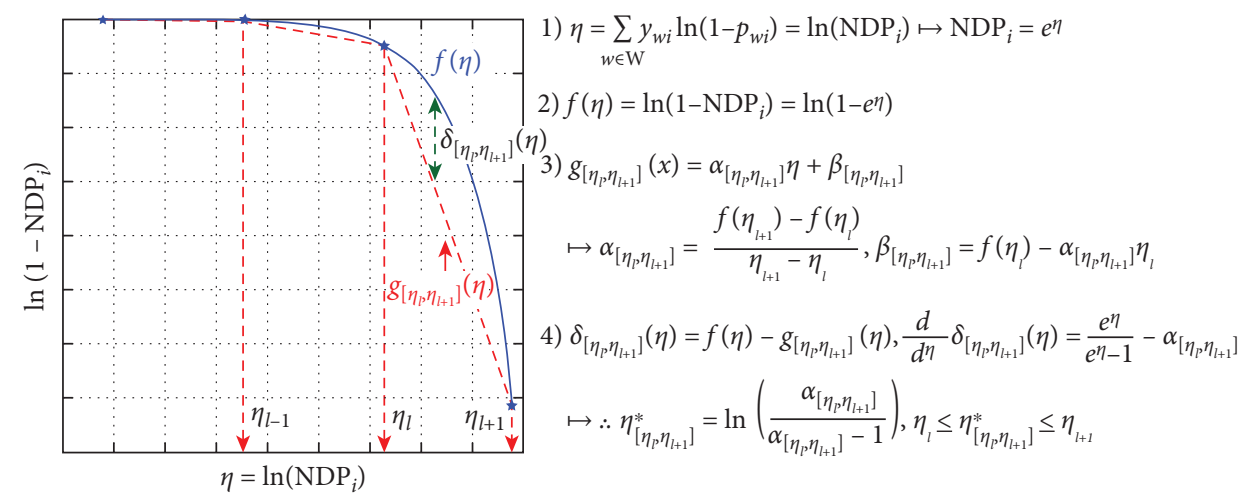

FIgURE 3: Error of the piecewise linear approximation.

TABLe 3: Notations for the piecewise linear approximation model.

\begin{tabular}{lc}
\hline Indices & Description \\
\hline$f(\eta)$ & Original function relating $(\eta)=\ln (\mathrm{NDP})$ and \\
$\eta_{l}$ & $f(\eta)=\ln (1-\mathrm{NDP})$ \\
$\left.g_{\left[\eta_{l}, \eta_{l}+1\right)}\right)$ & QUOTE $1 l^{\text {th }}$ breakpoint; $l \in\{0,1, \ldots, L\}$ \\
$\alpha_{\left[\eta_{l}, \eta_{l}+1\right)}$ & Piecewise linear function in interval $\left[\eta_{l}, \eta_{l}+1\right)$ \\
$\beta_{\left[\eta_{l}, \eta_{l}+1\right)}(\eta)$ & Slope of $g_{\left[\eta_{l}, \eta_{l}+1\right)}$ in interval $\left[\eta_{l}, \eta_{l}+1\right)$ \\
$\delta_{\left[\eta_{l}, \eta_{l}+1\right)}$ & Intersection of $g_{\left[\eta_{l}, \eta_{l+1}\right)}$ in interval $\left[\eta_{l}, \eta_{l}+1\right)$ \\
$\delta_{\left[\eta_{l}, \eta_{l}+1\right)}^{*}$ & Approximation error at $\eta$ in interval $\left[\eta_{l}, \eta_{l}+1\right)$ \\
\hline
\end{tabular}

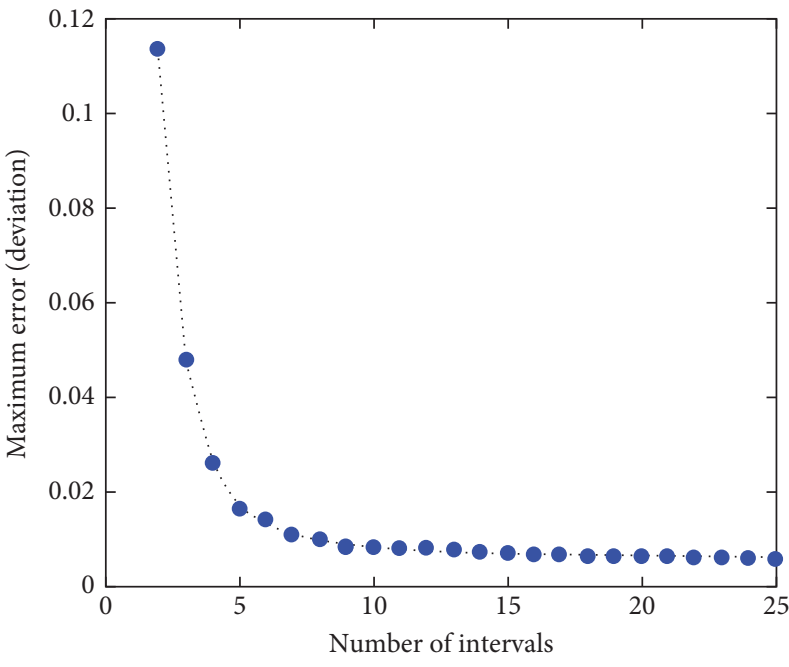

(a)

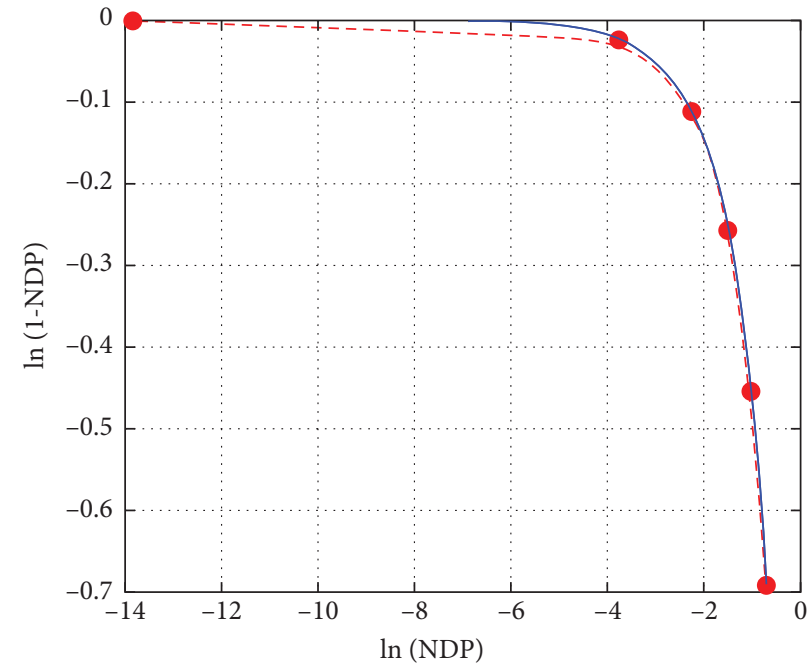

(b)

FIgURE 4: Continued. 


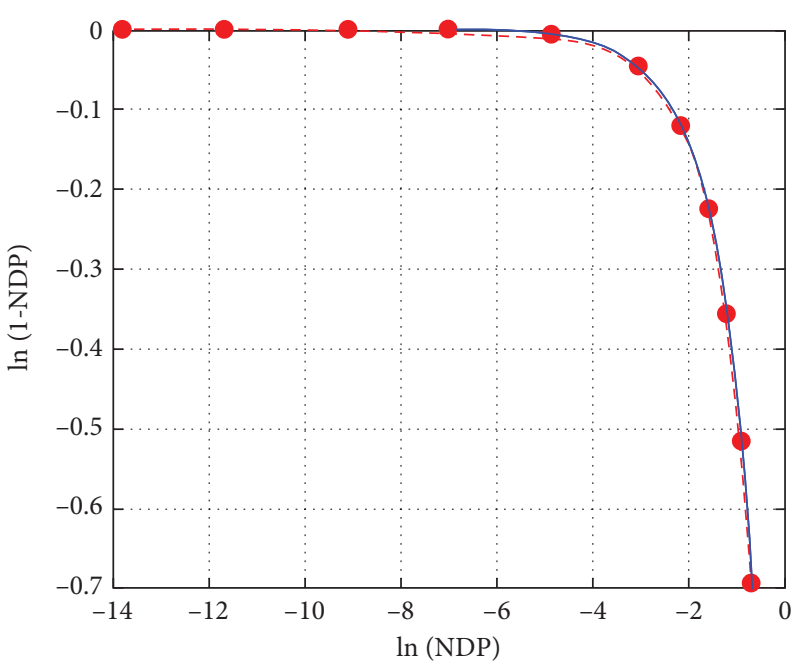

(c)

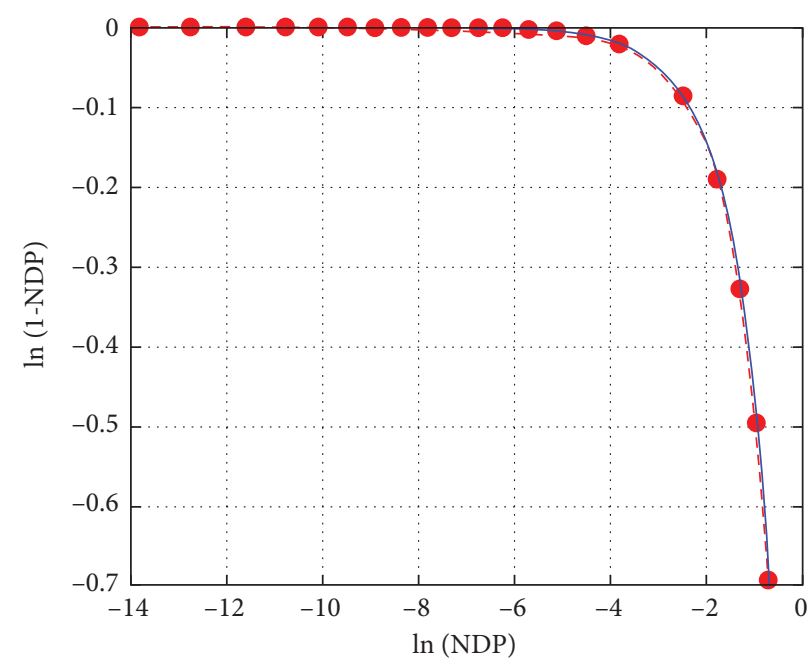

(d)

FiguRE 4: Optimal breakpoints for the piecewise linear approximation. (a) Error by the number of intervals. (b) Breakpoints for 5 intervals. (c) Breakpoints for 10 intervals. (d) Breakpoints for 20 intervals.

TABLE 4: Optimal breakpoints by the number of intervals.

\begin{tabular}{|c|c|c|c|c|}
\hline \multirow{2}{*}{ No. (l) } & \multicolumn{2}{|c|}{10 intervals } & \multicolumn{2}{|c|}{15 intervals } \\
\hline & $\eta_{l}$ & $\alpha_{(l, l+1)}$ & $\eta_{l}$ & $\alpha_{(l, l+1)}$ \\
\hline 0 & & $\cong 0.0$ & & $\cong 0.0$ \\
\hline 1 & -13.815511 & -0.000003 & -13.815511 & -0.000002 \\
\hline 2 & -11.709473 & -0.000039 & -12.372611 & -0.000010 \\
\hline 3 & -9.105682 & -0.000378 & -10.811520 & -0.000038 \\
\hline 4 & -7.009790 & -0.003195 & -9.668562 & -0.000109 \\
\hline 5 & -4.861735 & -0.022250 & -8.669014 & -0.000286 \\
\hline 6 & -3.066245 & -0.081970 & -7.721618 & -0.000738 \\
\hline 7 & -2.171228 & -0.182837 & -6.775789 & -0.001909 \\
\hline 8 & -1.599924 & -0.331971 & -5.825690 & -0.005137 \\
\hline 9 & -1.200840 & -0.539911 & -4.812854 & -0.015547 \\
\hline 10 & -0.909949 & -0.821355 & -3.658917 & -0.048623 \\
\hline 11 & -0.693147 & & -2.587288 & -0.119665 \\
\hline 12 & & & -1.929385 & -0.226607 \\
\hline 13 & & & -1.473317 & -0.375639 \\
\hline 14 & & & -1.139050 & -0.575393 \\
\hline 15 & & & -0.886755 & -0.837440 \\
\hline 16 & & & -0.693147 & \\
\hline
\end{tabular}

${ }^{*}$ When $\ln \mathrm{NDP}_{i}<\ln \left(10^{-6}\right), \alpha_{(0,1)} \cong 0.0$.

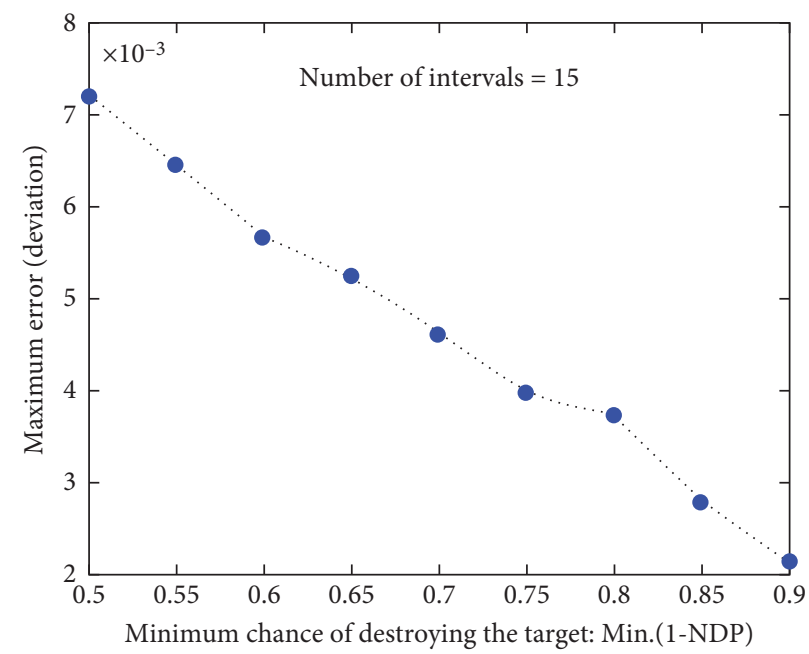

Figure 5: Approximation error by range Min.1-NDP, 0.999999. 
TABLE 5: Input parameters.

\begin{tabular}{|c|c|c|c|c|c|c|c|}
\hline \multirow{2}{*}{ Target $\left(T_{i}\right)$} & \multicolumn{2}{|c|}{ Coordinate } & \multirow{2}{*}{$V_{i}$} & \multicolumn{3}{|c|}{$p_{w i}$} & \multirow{2}{*}{ Other parameters } \\
\hline & $X$ & Y & & $w_{1}$ & $w_{2}$ & $w_{3}$ & \\
\hline$T_{1}$ & 62 & 97 & 0.83 & 0.62 & 0.59 & 0.56 & \\
\hline$T_{2}$ & 50 & 172 & 0.99 & 0.60 & 0.57 & 0.54 & \\
\hline$T_{3}$ & -76 & 169 & 0.43 & 0.73 & 0.69 & 0.66 & (i) $d_{i j}:=$ Euclidean distance between targets $i$ and $j$. \\
\hline$T_{4}$ & 5 & 178 & 0.84 & 0.62 & 0.59 & 0.56 & (ii) $v_{s}=[500,450,400]$ \\
\hline$T_{5}$ & -35 & 126 & 0.43 & 0.73 & 0.69 & 0.66 & (iii) $\overline{F T_{s}}=1.0(\forall s)$, and $\overline{S T_{k}}=8(\forall k)$. \\
\hline$T_{6}^{3}$ & 9 & 145 & 0.99 & 0.60 & 0.57 & 0.54 & (iv) $\overline{W_{i}}=3(\forall i)$, and $\overline{Q_{w}}=15(\forall w)$ \\
\hline$T_{7}$ & -20 & 193 & 0.86 & 0.62 & 0.59 & 0.56 & {$\left[\begin{array}{lll}2 & 2 & 0\end{array}\right] \quad\left[\begin{array}{lll}4 & 4 & 0\end{array}\right]$} \\
\hline$T_{8}$ & -17 & 117 & 0.59 & 0.67 & 0.64 & 0.60 & (v) $\left.J_{s k}=\mid \begin{array}{lll}0 & 2 & 2\end{array}\right], q_{s w}=\left|\begin{array}{lll}0 & 4 & 4\end{array}\right|$ \\
\hline$T_{9}$ & -64 & 59 & 0.81 & 0.62 & 0.59 & 0.56 & {$\left[\begin{array}{lll}2 & 0 & 2\end{array}\right] \quad\left[\begin{array}{lll}4 & 0 & 4\end{array}\right]$} \\
\hline$T_{10}$ & -49 & 180 & 0.64 & 0.66 & 0.63 & 0.59 & (vi) $P_{s i j}=\left(H_{s} /\left(H_{s}+d_{i j}\right)\right) ; H_{s}=[2500,2000,1500]$. \\
\hline
\end{tabular}

${ }^{*}$ Position of base: $(0,0)$.

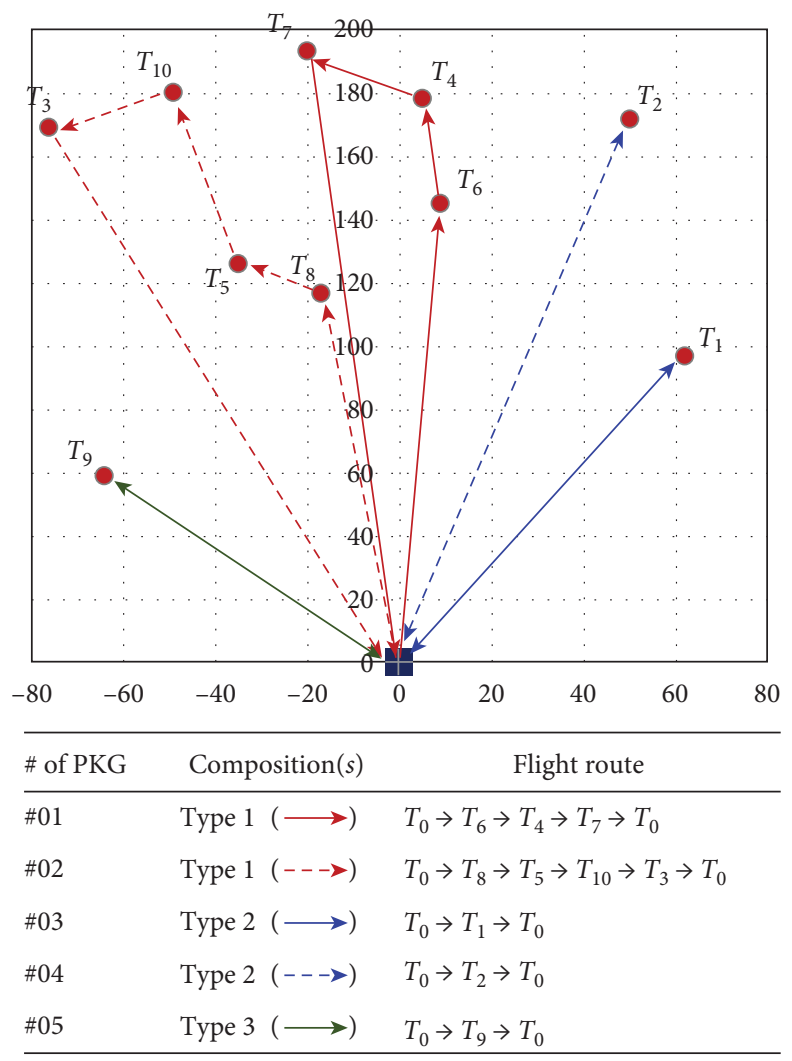

FIGURE 6: Flight routes of strike packages.

Table 6: Assigned weapons for each target.

\begin{tabular}{lcccccccccc}
\hline Target & $T_{1}$ & $T_{2}$ & $T_{3}$ & $T_{4}$ & $T_{5}$ & $T_{6}$ & $T_{7}$ & $T_{8}$ & $T_{9}$ & $T_{10}$ \\
\hline 10 intervals & $w_{2}(1) w_{3}(2)$ & $w_{3}(3)$ & $\mathbf{w}_{1}(\mathbf{1}) \mathbf{w}_{2}(\mathbf{1})$ & $w_{1}(2)$ & $\mathbf{w}_{2}(2)$ & $w_{2}(3)$ & $w_{1}(2)$ & $\mathbf{w}_{1}(\mathbf{1}) \mathbf{w}_{2}(\mathbf{1})$ & $w_{3}(3)$ & $\mathbf{w}_{1}(\mathbf{2})$ \\
15 intervals & $w_{2}(1) w_{3}(2)$ & $w_{3}(3)$ & $\mathbf{w}_{1}(2)$ & $w_{1}(2)$ & $\mathbf{w}_{1}(2)$ & $w_{2}(3)$ & $w_{1}(2)$ & $\mathbf{w}_{2}(2)$ & $w_{3}(3)$ & $\mathbf{w}_{2}(2)$ \\
\hline
\end{tabular}

*Weapon: type (quantity).

targets is $0.86 \%$ at the maximum and $0.07 \%$ at the minimum. In the numerical experiment applying the piecewise linear function with 15 intervals, the objective function's error is estimated as $0.26 \%$, and the error of MSI at the targets is $0.66 \%$ at the maximum and $0.03 \%$ at the minimum. The disparity in the MSIs' errors by the piecewise linear functions alters the weapon assignment for some targets. However, considering the real values, that is, 6.2029 
TABLe 7: Analysis of the piecewise linear approximation.

\begin{tabular}{|c|c|c|c|c|c|c|c|c|c|c|c|c|}
\hline \multicolumn{2}{|c|}{ Class. } & $T_{1}$ & $T_{2}$ & $T_{3}$ & $T_{4}$ & $T_{5}$ & $T_{6}$ & $T_{7}$ & $T_{8}$ & $T_{9}$ & $T_{10}$ & Sum \\
\hline \multicolumn{13}{|c|}{ (a) Number of intervals $(L)=10$} \\
\hline \multirow{6}{*}{ MILP } & $\ln \left(V_{i}\right)$ & -0.1863 & -0.0101 & -0.8440 & -0.1744 & -0.8440 & -0.0101 & -0.1508 & -0.5276 & -0.2107 & -0.4463 & \\
\hline & $\mathrm{LCP}_{i}$ & -0.0560 & -0.0858 & -0.0879 & -0.0697 & -0.0542 & -0.0565 & -0.0813 & -0.0462 & -0.0564 & -0.0763 & \\
\hline & $\ln \left(1-\mathrm{NDP}_{i}\right)$ & -0.0914 & -0.1081 & -0.0957 & -0.1642 & -0.1071 & -0.0915 & -0.1642 & -0.1286 & -0.0972 & -0.1236 & \\
\hline & $1-\mathrm{NDP}_{i}$ & 0.9127 & 0.8975 & 0.9087 & 0.8485 & 0.8985 & 0.9125 & 0.8485 & 0.8794 & 0.9074 & 0.8838 & \\
\hline & Equation (21) & -0.3337 & -0.2039 & -1.0276 & -0.4083 & -1.0052 & -0.1581 & -0.3964 & -0.7024 & -0.3643 & -0.6462 & -5.2460 \\
\hline & $e^{\mathrm{Eq} \cdot(21)}$ & 0.7163 & 0.8155 & 0.3579 & 0.6648 & 0.3660 & 0.8538 & 0.6728 & 0.4954 & 0.6947 & 0.5241 & 6.1611 \\
\hline \multirow{4}{*}{ Real values } & $V_{i}$ & 0.83 & 0.99 & 0.43 & 0.84 & 0.43 & 0.99 & 0.86 & 0.59 & 0.81 & 0.64 & \\
\hline & $\mathrm{CP}_{i}$ & 0.9456 & 0.9178 & 0.9159 & 0.9327 & 0.9472 & 0.9451 & 0.9219 & 0.9548 & 0.9452 & 0.9265 & \\
\hline & $1-\mathrm{NDP}_{i}$ & 0.9206 & 0.9027 & 0.9163 & 0.8556 & 0.9039 & 0.9205 & 0.8556 & 0.8812 & 0.9148 & 0.8844 & \\
\hline & Equation (1) & 0.7225 & 0.8202 & 0.3609 & 0.6703 & 0.3682 & 0.8612 & 0.6784 & 0.4964 & 0.7004 & 0.5244 & 6.2029 \\
\hline \multicolumn{2}{|l|}{$\begin{array}{l}\text { Error rate of } \\
\text { Obj. Func. }\end{array}$} & $0.86 \%$ & $0.57 \%$ & $0.83 \%$ & $0.83 \%$ & $0.60 \%$ & $0.86 \%$ & $0.83 \%$ & $0.21 \%$ & $0.81 \%$ & $0.07 \%$ & $0.67 \%$ \\
\hline \multicolumn{13}{|c|}{ (b) Number of intervals $(L)=15$} \\
\hline \multirow{6}{*}{ MILP } & $\ln \left(V_{i}\right)$ & -0.1863 & -0.0101 & -0.8440 & -0.1744 & -0.8440 & -0.0101 & -0.1508 & -0.5276 & -0.2107 & -0.4463 & \\
\hline & $\mathrm{LCP}_{i}$ & -0.0560 & -0.0858 & -0.0879 & -0.0697 & -0.0542 & -0.0565 & -0.0813 & -0.0462 & -0.0564 & -0.0763 & \\
\hline & $\ln \left(1-\mathrm{NDP}_{i}\right)$ & -0.0846 & -0.1090 & -0.0767 & -0.1562 & -0.0767 & -0.0848 & -0.1562 & -0.1433 & -0.0931 & -0.1499 & \\
\hline & $1-\mathrm{NDP}_{i}$ & 0.9188 & 0.8967 & 0.9262 & 0.8554 & 0.9262 & 0.9187 & 0.8554 & 0.8665 & 0.9111 & 0.8608 & \\
\hline & Equation (21) & -0.3269 & -0.2049 & -1.0085 & -0.4003 & -0.9749 & -0.1514 & -0.3884 & -0.7171 & -0.3602 & -0.6724 & -5.2050 \\
\hline & $e^{\text {Eq. }(21)}$ & 0.7211 & 0.8148 & 0.3648 & 0.6701 & 0.3772 & 0.8595 & 0.6782 & 0.4881 & 0.6975 & 0.5105 & 6.1819 \\
\hline \multirow{4}{*}{ Real values } & $V_{i}$ & 0.83 & 0.99 & 0.43 & 0.84 & 0.43 & 0.99 & 0.86 & 0.59 & 0.81 & 0.64 & \\
\hline & $\mathrm{CP}_{i}$ & 0.9456 & 0.9178 & 0.9159 & 0.9327 & 0.9472 & 0.9451 & 0.9219 & 0.9548 & 0.9452 & 0.9265 & \\
\hline & $1-\mathrm{NDP}_{i}$ & 0.9206 & 0.9027 & 0.9271 & 0.8556 & 0.9271 & 0.9205 & 0.8556 & 0.8704 & 0.9148 & 0.8631 & \\
\hline & Equation (1) & 0.7225 & 0.8202 & 0.3651 & 0.6703 & 0.3776 & 0.8612 & 0.6784 & 0.4903 & 0.7004 & 0.5118 & 6.1979 \\
\hline $\begin{array}{l}\text { Error rate of } \\
\text { Obj. Func. }\end{array}$ & & $0.19 \%$ & $0.66 \%$ & $0.10 \%$ & $0.03 \%$ & $0.10 \%$ & $0.20 \%$ & $0.03 \%$ & $0.45 \%$ & $0.40 \%$ & $0.26 \%$ & $0.26 \%$ \\
\hline
\end{tabular}

and 6.1979, of the objective function in two experiments, the weapon assignment change effect is insignificant. Furthermore, the overall approximation error of the objective function is not represented as the maximum or minimum error in the targets, and it is the average level of the errors in the targets. That is, the objective function's error does not appear as the accumulation of each target's MSI error. Thus, we could expect that overall approximation error is maintained even if the number of targets in a problem increases.

\section{Conclusions and Future Studies}

This study focuses on the optimization problem to establish an effective air operation plan considering critical operational factors, such as the attack effect on the targets and the strike packages' survivability. The MILP model proposed in this study is expected to improve the efficiency of the decision-making process for an air operation because it simultaneously provides various information for composing strike packages, such as the composition type, assigned targets, flight route, and weapons for each target. In addition, the initial mathematical model for the original problem is developed as an MINLP model, including nonlinear functions for evaluating MSI and the survivability of strike packages. However, there is no efficient algorithm for searching for the optimal solution of a nonlinear programming model in modern theory. Thus, the MINLP model is converted to a MILP model by the logarithmic transformation and a piecewise linear approximation method. The optimization methodology for the piecewise linear function to approximate a nonlinear function could be applied to various fields such as reliability engineering and the optimization for a supply chain with uncertainty.

Future studies could include the following: First, the MSI for future warfare should be developed considering the concept of future warfare, battlefield space, combat means, and combat type. The second issue is to create a highly reliable algorithm to provide a reasonable solution faster, and it contributes to reducing the decision-making time. The solution method could be developed by various algorithms, such as metaheuristic algorithms and reinforcement learning algorithms. For enhancing its reliability, strategies such as parallelization and hybridization of algorithms could also be employed. The final topic is to apply this study's contents to optimization problems reflecting the uncertainty of the real world. For example, it can be applied to supply chain design to prepare for and respond to a disaster with high uncertainty.

\section{Data Availability}

The data for verifying the results of this study and for followup studies are presented in Tables 4-7 of this article.

\section{Conflicts of Interest}

The author declares that there are no conflicts of interest regarding the publication of this paper. 


\section{Acknowledgments}

This work was supported by the National Research Foundation of Korea (Grant no. 2018R1C1B5043513) and Advanced Materials and Components Laboratory Project for Defense Industry (Grant no. DCL2020 L).

\section{References}

[1] A. S. Manne, "A target-assignment problem," Operations Research, vol. 6, no. 3, pp. 346-351, 1958.

[2] M. T. Davis, M. J. Robbins, and B. J. Lunday, "Approximate dynamic programming for missile defense interceptor fire control," European Journal of Operational Research, vol. 259, no. 3, pp. 873-886, 2017.

[3] H. Xu, Q. Xing, and Z. Tian, "MOQPSO-D/S for air and missile defense WTA problem under uncertainty," Mathematical Problems in Engineering, vol. 2017, Article ID 9897153, 10 pages, 2017.

[4] X. Hu, P. Luo, X. Zhang, and J. Wang, "Improved ant colony optimization for weapon-target assignment," Mathematical Problems in Engineering, vol. 2, 2018.

[5] O. Karasakal, N. E. Özdemirel, and L. Kandiller, "Anti-ship missile defense for a naval task group," Naval Research Logistics (NRL), vol. 58, no. 3, pp. 304-321, 2011.

[6] Z. J. Lee, S. F. Su, and C. Y. Lee, "Efficiently solving general weapon-target assignment problem by genetic algorithms with greedy eugenics," IEEE Transactions on Systems, Man, and Cybernetics, Part B (Cybernetics), vol. 33, no. 1, pp. 113-121, 2003.

[7] N. Jia, Z. Yang, and K. Yang, "Operational effectiveness evaluation of the swarming UAV s combat system based on a system dynamics model," IEEE Access, vol. 7, pp. 2520925224, 2019.

[8] P. Zhao, J. Wang, and L. Kong, "Decentralized algorithms for weapon-target assignment in swarming combat system," Mathematical Problems in Engineering, vol. 2019, pp. 1-15, 2019.

[9] L. H. Wegner, The "special Weighted Distribution Problem" of Linear Programming: An Application and a FORTRAN Program, Rand Corporation, Santa Monica, CL, USA, 1966.

[10] B. J. Griggs, "An air mission planning algorithm for a theater level combat model," Master's Thesis, Department of Science Operations Research, Air Force Institute of Technology, Washington, DC, USA, 1994.

[11] D. R. Castro, "Optimization models for allocation of air strike assets with persistence," Master's Thesis, Department of Operations Research, Naval Postgraduate School, Monterey, CA, USA, 2002.

[12] D. R. Lee and J. H. Yang, "The optimal allocation of aircrafts to targets by using mixed integer programming," Korean Management Science Review, vol. 25, no. 1, pp. 55-74, 2008.

[13] B. J. Jeong and C. Y. Kim, "Aircraft allocation model: application of the goal programming," Military Operations Research Society of Korea, vol. 20, no. 1, pp. 49-79, 1994.

[14] B. J. Griggs, G. S. Parnell, and L. J. Lehmkuhl, “An air mission planning algorithm using decision analysis and mixed integer programming," Operations Research, vol. 45, no. 5, pp. 662-676, 1997.

[15] V. C. Li, G. L. Curry, and E. A. Boyd, "Towards the real time solution of strike force asset allocation problems," Computers and Operations Research, vol. 31, no. 2, pp. 273-291, 2004.

[16] J. M. Rosenberger, H. S. Hwang, R. P. Pallerla, A. Yucel, R. L. Wilson, and E. G. Brungardt, The Generalized Weapon
Target Assignment Problem, Texas Univ at Arlington, Arlington, TX, USA, 2005.

[17] D. Ahner and C. Parson, "Weapon tradeoff analysis using dynamic programming for a dynamic weapon target assignment problem within a simulation," in Proceedings of the 2013 Winter Simulations Conference (WSC), pp. 2831-2841, Washington, DC, USA, December 2013.

[18] C. R. Parson, Approximate dynamic programming for military resource allocation, Ph.D. Thesis, Air Force Institute of Technology, Bengaluru, India, 2014.

[19] S. P. Lloyd and H. S. Witsenhausen, "Weapons allocation is NP-complete," in Proceedings of the 1986 Summer Computer Simulation Conference, pp. 1054-1058, Reno, NV, USA, July 1986.

[20] S. C. Chang, R. M. James, and J. J. Shaw, "Assignment algorithm for kinetic energy weapons in boost phase defence," in Proceedings of the 26th IEEE Conference on Decision and Control, pp. 1678-1683, Honolulu, HW, USA, September 1987.

[21] D. Orlin, "Optimal weapons allocation against layered defenses," Naval Research Logistics (NRL), vol. 34, no. 5, pp. 605-617, 1987.

[22] J. Lee and M. Shin, "Stochastic weapon target assignment problem under uncertainty in targeting accuracy," Journal of Korea Operations Research and Management Science, vol. 41, no. 3, pp. 23-36, 2016.

[23] O. Kwon, D. Kang, K. Lee, and S. Park, "Lagrangian relaxation approach to the targeting problem," Naval Research Logistics (NRL), vol. 46, no. 6, pp. 640-653, 1999.

[24] R. K. Ahuja, A. Kumar, K. C. Jha, and J. B. Orlin, "Exact and heuristic algorithms for the weapon-target assignment problem," Operations Research, vol. 55, no. 6, pp. 1136-1146, 2007.

[25] Y. Owechko and S. Shams, "Comparison of neural network and genetic algorithms for a resource allocation problem," IEEE International Conference on Neural Networks (ICNN'94), vol. 7, pp. 4655-4660, 1994.

[26] Z. J. Lee, S. F. Su, and C. Y. Lee, "A genetic algorithm with domain knowledge for weapon-target assignment problems," Journal of the Chinese Institute of Engineers, vol. 25, no. 3, pp. 287-295, 2002a.

[27] H. Lu, H. Zhang, X. Zhang, and R. Han, "An improved genetic algorithm for target assignment, optimization of naval fleet air defense," in Proceedings of the 2006 6th IEEE World Congress on Intelligent Control and Automation, pp. 3401-3405, Dalian, China, May 2006.

[28] P. Li, L. Wu, and F. Lu, "A mutation-based ga for weapontarget allocation problem subject to spatial constraints," in Proceedings of the 2009 IEEE International Workshop on Intelligent Systems and Applications, pp. 1-4, Pisa, Italy, December 2009.

[29] Z. J. Lee, C. Y. Lee, and S. F. Su, “An immunity-based ant colony optimization algorithm for solving weapon-target assignment problem," Applied Soft Computing, vol. 2, no. 1, pp. 39-47, 2002.

[30] W. Yanxia, Q. Longjun, G. Zhi, and M. Lifeng, "Weapon target assignment problem satisfying expected damage probabilities based on ant colony algorithm," Journal of Systems Engineering and Electronics, vol. 19, no. 5, pp. 939944, 2008.

[31] L. Jinjun, C. Rong, and X. Jiguangt, "Dynamic WTA optimization model of air defense operation of warships' formation," Journal of Systems Engineering and Electronics, vol. 17, no. 1, pp. 126-131, 2006. 
[32] H. Naeem and A. Masood, "An optimal dynamic threat evaluation and weapon scheduling technique," KnowledgeBased Systems, vol. 23, no. 4, pp. 337-342, 2010.

[33] A. Tokgöz and S. Bulkan, "Weapon target assignment with combinatorial optimization techniques," International Journal of Advanced Research in Artificial Intelligence, vol. 2, no. 7, pp. 39-50, 2013.

[34] D. L. Luo, C. L. Shen, B. Wang, and W. H. Wu, "Air combat decision-making for cooperative multiple target attack using heuristic adaptive genetic algorithm," in Proceedings of the 2005 IEEE International Conference on Machine Learning and Cybernetics, pp. 473-478, Guangzhou, China, August 2005.

[35] G. Shang, Z. Zaiyue, Z. Xiaoru, and C. Cungen, "Immune genetic algorithm for weapon-target assignment problem," in Proceedings of the IEEE Workshop on Intelligent Information Technology Application (IITA 2007), pp. 145-148, Zhang Jiajia, China, December 2007.

[36] S. Zhihua, F. Zhu, and Z. Duolin, "A heuristic genetic algorithm for solving constrained Weapon-Target Assignment problem," in Proceedings of the IEEE International Conference on Intelligent Computing and Intelligent Systems, pp. 336-341, Shanghai, China, November 2009.

[37] W. A. Menner, "The navy's tactical aircraft strike planning process," Johns Hopkins APL Technical Digest, vol. 18, no. 1, pp. 90-104, 1997.

[38] I. Kara, G. Laporte, and T. Bektas, "A note on the lifted Miller-Tucker-Zemlin subtour elimination constraints for the capacitated vehicle routing problem," European Journal of Operational Research, vol. 158, no. 3, pp. 793-795, 2004.

[39] U. Pferschy and R. Staněk, "Generating subtour elimination constraints for the TSP from pure integer solutions," Central European Journal of Operations Research, vol. 25, no. 1, pp. 231-260, 2017. 